\title{
Induction and Activation by Zinc of NADPH Oxidase in Cultured Cortical Neurons and Astrocytes
}

\author{
Kyung-Min Noh and Jae-Young Koh \\ National Creative Research Initiative Center for the Study of CNS Zinc and Department of Neurology, University of Ulsan \\ College of Medicine, Seoul 138-736, Korea
}

Zinc overload may be a key mechanism of neuronal death in acute brain injury. We have demonstrated previously that zinc overload neurotoxicity involves protein kinase $C$ (PKC)dependent rises in intracellular levels of reactive oxygen species (ROS). However, the cascade linking PKC activation to ROS generation in cultured cortical neurons has been unknown. A recent study has demonstrated that ROS-generating NADPH oxidase is present in sympathetic neurons and contributes to NGF deprivation-induced cell death. Because NADPH oxidase is activated by PKC, in the present study, we examined the possibility that NADPH oxidase is the effector for oxidative stress in zinc-overloaded cortical cells.

Reverse transcription-PCR and Western blot analyses revealed that naive cultured cortical cells express subunits of NADPH oxidase at low levels. Exposure to zinc substantially increased levels of NADPH oxidase subunits in both neurons and astrocytes. In addition, zinc exposure induced transloca-

Recent evidence indicates that endogenous zinc may play a key role in neuronal death after acute brain insults such as ischemia, seizures, and trauma (Frederickson et al., 1989; Tonder et al., 1990; Koh et al., 1996; Choi and Koh, 1998; Suh et al., 2000). In these brain injury paradigms, intracellular accumulation of zinc correlates well with neuronal death at the single cell level. Furthermore, chelation of zinc prevents both intracellular zinc accumulation and neuronal death in those brain injury models. Cytotoxic mechanisms of intracellular zinc overload may involve diverse processes such as mitochondrial damage, nicotinamide adenine dinucleotide (NAD)-positive $\left(\mathrm{NAD}^{+}\right)$degradation, ATP depletion, and caspase activation (Lobner et al., 1997; Manev et al., 1997; Sheline et al., 2000). In addition, evidences suggest that oxidative stress plays a significant role in zinc neurotoxicity in cortical culture. First, zinc influx increases the levels of reactive oxygen species (ROS) in neurons (Kim et al., 1999; Sensi et al., 1999). Second, various neuroprotective measures effective against zinc toxicity, such as protein kinase $\mathrm{C}$ (PKC) inhibitors, attenuate zinc-induced increases in ROS in parallel (Noh et al., 1999). Finally, antioxidants attenuate zinc neurotoxicity (Kim et al., 1999). Although the evidence indicates that oxidative stress is a significant mechanism of zinc-induced neuronal death, the effector protein(s) directly responsible for the generation of ROS in zinc-injured cells is unknown.

Received June 26, 2000; revised Aug. 21, 2000; accepted Sept. 26, 2000.

This study was supported by the National Creative Research Initiatives of Korean Ministry of Science and Technology (J.-Y.K.).

Correspondence should be addressed to Dr. Jae-Young Koh, National Creative Research Initiative Center for the Study of CNS Zinc, and Department of Neurology, University of Ulsan College of Medicine, 388-1 Poongnap-Dong Songpa-Gu Seoul 138-736, Korea. E-mail: jkko@www.amc.seoul.kr.

Copyright (C) 2000 Society for Neuroscience $0270-6474 / 00 / 200001-05 \$ 15.00 / 0$ tion of the $\mathrm{p} 47^{\mathrm{PHOX}}$ and $\mathrm{p} 67^{\mathrm{PHOX}}$ subunits to the membrane, a signature event for NADPH oxidase activation. Addition of a selective PKC inhibitor, GF109203X, blocked both the induction and the membrane translocation of NADPH oxidase by zinc. Supporting the role for NADPH oxidase in zinc-triggered oxidative injury, NADPH oxidase inhibitors attenuated ROS production and cortical neuronal death induced by zinc. In addition, $\mathrm{Cu} / \mathrm{Zn}$-superoxide dismutase and catalase attenuated zinc-induced cortical neuronal death.

Our results have demonstrated that zinc overload induces and activates NADPH oxidase in cortical neurons and astrocytes in a PKC-dependent manner. Thus, NADPH oxidase may be an enzyme contributing to ROS generation in zincoverloaded cortical neurons and astrocytes.

Key words: oxidative stress; protein kinase C; superoxide; astrocyte; calcium; neuronal death

NADPH oxidase is a superoxide-producing enzyme consisting of the membrane (gp91 ${ }^{\text {PHOX }}$ and $\mathrm{p} 22^{\text {PHOX }}$ ) and the cytosolic (p47 ${ }^{\text {PHOX }}, \mathrm{p} 67^{\text {PHOX }}$, and $\mathrm{p} 40^{\text {PHOX }}$ ) components (DeLeo and Quinn, 1996; Babior, 1999). In addition, small G-proteins such as rac1, as well as kinases including PKC, regulate its activity (Heinecke et al., 1990; Benna et al., 1997; Reeves et al., 1999; Ozaki et al., 2000). Although NADPH oxidase is mainly expressed in phagocytic cells, an increasing body of evidence suggests that various subunits of NADPH oxidase are also expressed in nonphagocytic cells such as mesangial cells, endothelial cells, vascular smooth muscles, and fibroblasts (Fukui et al., 1995; Jones et al., 1995, 1996; Thannickal and Fanburg, 1995). However, functions of NADPH oxidase subunits in nonphagocytic cells are not yet clearly delineated. Recently, all of the subunits of NADPH oxidase are to be expressed by sympathetic ganglion neurons and to contribute to NGF-deprivation-induced ROS generation and cell death, suggesting the possibility that neurons in general might express NADPH oxidase (Tammariello et al., 2000). Because NADPH oxidase can be activated by PKC and because PKC

This article is published in The Journal of Neuroscience, Rapid Communications Section, which publishes brief, peer-reviewed papers online, not in print. Rapid Communications are posted online approximately one month earlier than they would appear if printed. They are listed in the Table of Contents of the next open issue of JNeurosci. Cite this article as: JNeurosci, 2000, 20:RC111 (1-5). The publication date is the date of posting online at www.jneurosci.org.

http://www.jneurosci.org/cgi/content/full/4737 
activation appears to be a key step in zinc-induced oxidative injury, we hypothesized that NADPH oxidase may be the effector enzyme mediating the PKC-dependent oxidative injury in zincoverloaded cultured cortical neurons. This possibility was examined in the present study.

\section{MATERIALS AND METHODS}

Cortical cell culture and assessment of cell death. Mixed mouse cortical cultures containing both neurons and astrocytes, and pure astrocyte cultures, were prepared from fetal (15 d of gestation) and neonatal (1-3 postnatal days) mice, respectively, as described previously (Kim et al., 1999). Near-pure neuronal cultures were prepared from fetal mice (Sheline and Choi, 1998). Immunocytochemical staining with antiMacrophage (Mac-1-antigen) antibody (Boehringer Mannheim, Mannheim, Germany) revealed that $<3 \%$ of cells were microglia in all three types of cultures.

Brief (15 min) exposure of cortical cultures (10-13 d in vitro $)$ to zinc was performed in serum-free HBSS at room temperature. After exposure, cultures were incubated in serum-free MEM and placed back into the incubator. In addition to morphological assessment under a phasecontrast microscope, in most experiments, overall neuronal cell injury was quantitatively assessed by the measurement of lactate dehydrogenase (LDH) (Koh and Choi, 1987) released by damaged cells into the bathing medium $24 \mathrm{hr}$ after exposure to zinc. Each $\mathrm{LDH}$ value, after subtracting background LDH value in sham-washed controls, was scaled to the maximal neuronal LDH release (of 100) produced by $24 \mathrm{hr}$ exposure of sister cultures to $300 \mu \mathrm{M}$ NMDA, which produces near-complete neuronal death with no glial damage.

Reverse transcription-PCR. RNA was prepared with TRIZOL (Life Technologies, Gaithersburg, MD) and reverse-transcribed to cDNA using oligo-dT 14 primers (Promega, Madison, WI). Specific cDNAs were amplified for 30 cycles. Signals for amplified cDNAs, after separation on
$1.7 \%$ agarose gels, were visualized with ethidium bromide and quantitatively measured with an image analyzer (Alpha Imager 2000; Alpha Innotech, San Leandro, CA). Primer sequences were as follows: 5'CTTGGATGATAGCACTGCAC-3' and 5'-CTTCATCTGAAGCTCAATGG-3' for p91 ${ }^{\text {PHOX }}$ (626 bp); 5'-AGAGCGACTTTGAGCAGCTT-3' and 5'-TGTGGAGACACACCCTTGAT-3' for p40 ${ }^{\text {PHOX }}$ (460 bp); 5' -AGCCTGAGACATACCTGGTG-3' and 5' -AGACTTCTGCAGATACATGG-3' for p47 ${ }^{\text {PHOX }}$ (446 bp); 5'-CAAGGCTACGGTTGTAGCAT-3' and 5'-ACCTTGAGCATGTAAGGCAT-3' for p67 ${ }^{\text {PHOX }}$ (465 bp); and 5'-CTCAAGATTGTCAGCAATGC-3' and 5'-CACTGAGGACCAGGTTGTCT-3' for glyceraldehyde-3-phosphate dehydrogenase (415 bp).

\section{Cell lysis, fractionation, and immunoblotting}

Cortical cells were lysed in lysis buffer and centrifuged at 14,000 rpm for $20 \mathrm{~min}$. The pellet was discarded, and the supernatant was used for protein quantification. Fractionation of cytosol and membrane was performed as described previously (Noh et al., 1999). Equal amounts of protein from total cell lysates or membrane and cytosolic fraction were electrophoresed on $10 \%$ SDS-PAGE and transferred to polyvinylidene difluoride (PVDF) membranes. PVDF membranes were then incubated with respective primary antibodies (anti-p67 ${ }^{\text {PHOX }}$, anti-p47 ${ }^{\text {PHOX }}$, rac-1, and anti-gp91) overnight at $4^{\circ} \mathrm{C}$ The enhanced chemiluminescence (Amersham Pharmacia Biotech, Arlington Heights, IL) protocol was used to visualize the immunoreactive bands.

Immunohistochemistry. After fixation (4\% paraformaldehyde) and blocking, cells were double-labeled with anti-p67 ${ }^{\text {PHOX }}$ antibody (Transduction Laboratories, San Diego, CA) and either anti-glial fibrillary acidic protein (GFAP) or anti-microtubule-associated protein-2 (MAP2) antibody (Sigma, St. Louis, MO). After washes, signals were visualized by appropriate secondary antibodies coupled to either fluorescein isothiocyanate (FITC) or rhodamine (Jackson ImmunoResearch, West Grove, PA).
A

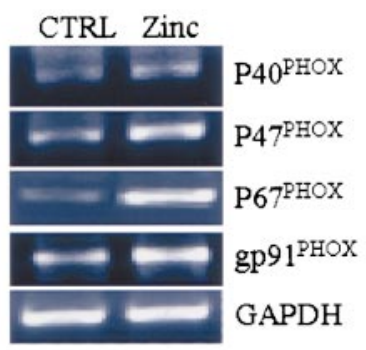

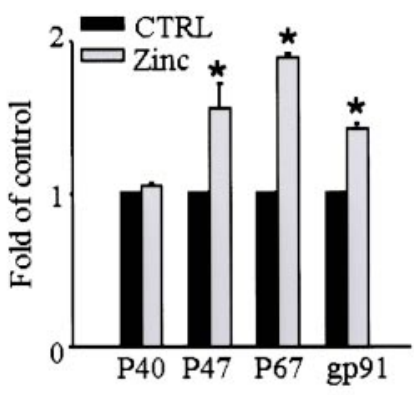

B
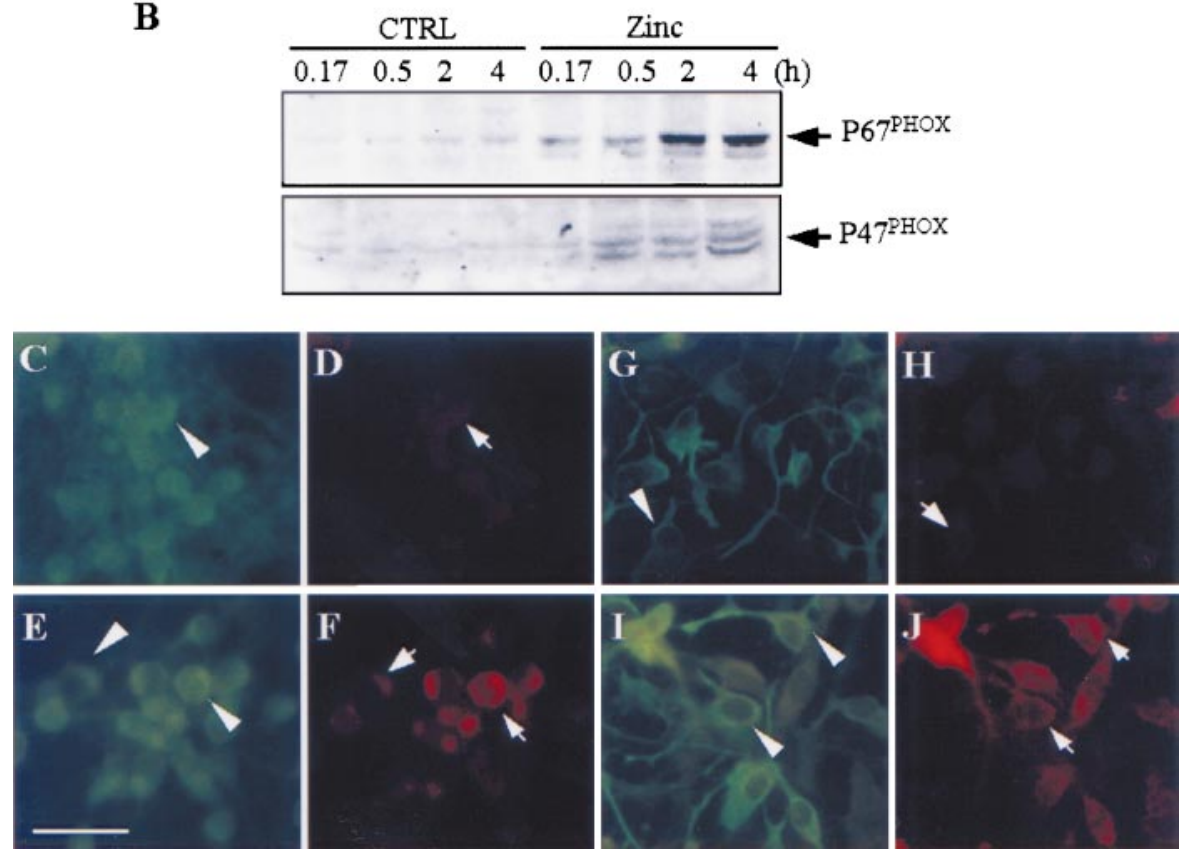

Figure 1. Induction of NADPH oxidase subunits in cortical cells exposed to zinc. $A$, RT-PCR for $\mathrm{p}^{\mathrm{PHOX}}, \mathrm{p} 47^{\text {PHOX }}, \mathrm{p} 67^{\text {PHOX }}$, and gp91 ${ }^{\text {PHOX }}$ in sham-washed control cultures (CTRL) and in sister cultures $2 \mathrm{hr}$ after $15 \mathrm{~min}$ exposure to 400 $\mu \mathrm{M}$ zinc (representative of 3 experiments). Bars denote densitometer readings of RT-PCR signals expressed as folds of respective control values (mean \pm SEM; $n=3$ ). Asterisks denote difference from respective controls $(p<0.05$; twotailed $t$ test). $B$, Western blots for $\mathrm{p} 67^{\mathrm{PHOX}}$ and $\mathrm{p} 47^{\text {PHOX }}$. Fifteen minute exposure to $400 \mu \mathrm{M}$ zinc increased both $067^{\mathrm{PHOX}}$ and $\mathrm{p} 47^{\mathrm{PHOX}}$ protein levels in cortical culture beginning $0.5-2 \mathrm{hr}$ after zinc exposure. $C-F$, Fluorescent photomicrographs of near-pure neuronal cultures, shamwashed control $(C, D)$, or $4 \mathrm{hr}$ after $15 \mathrm{~min}$ exposure to $400 \mu \mathrm{M}$ zinc $(E, F)$, stained with anti-MAP2 antibody $(C, E$, FITC) and then with anti-p67 ${ }^{\text {PHOX }}$ antibody ( $D, F$, rhodamine). $A r$ rows and arrowheads denote identical landmark cells in matched sets. $G-J$, Fluorescent microphotographs of astrocyte-rich cultures, shamwashed control $(G, H)$, or $4 \mathrm{hr}$ after $15 \mathrm{~min}$ exposure to $400 \mu \mathrm{M}$ zinc $(I, J)$, stained with anti-GFAP antibody $(G, I)$ and anti-p67 ${ }^{\text {PHOX }}$ antibody $(H, J)$. Scale bar, $50 \mu \mathrm{m}$. 


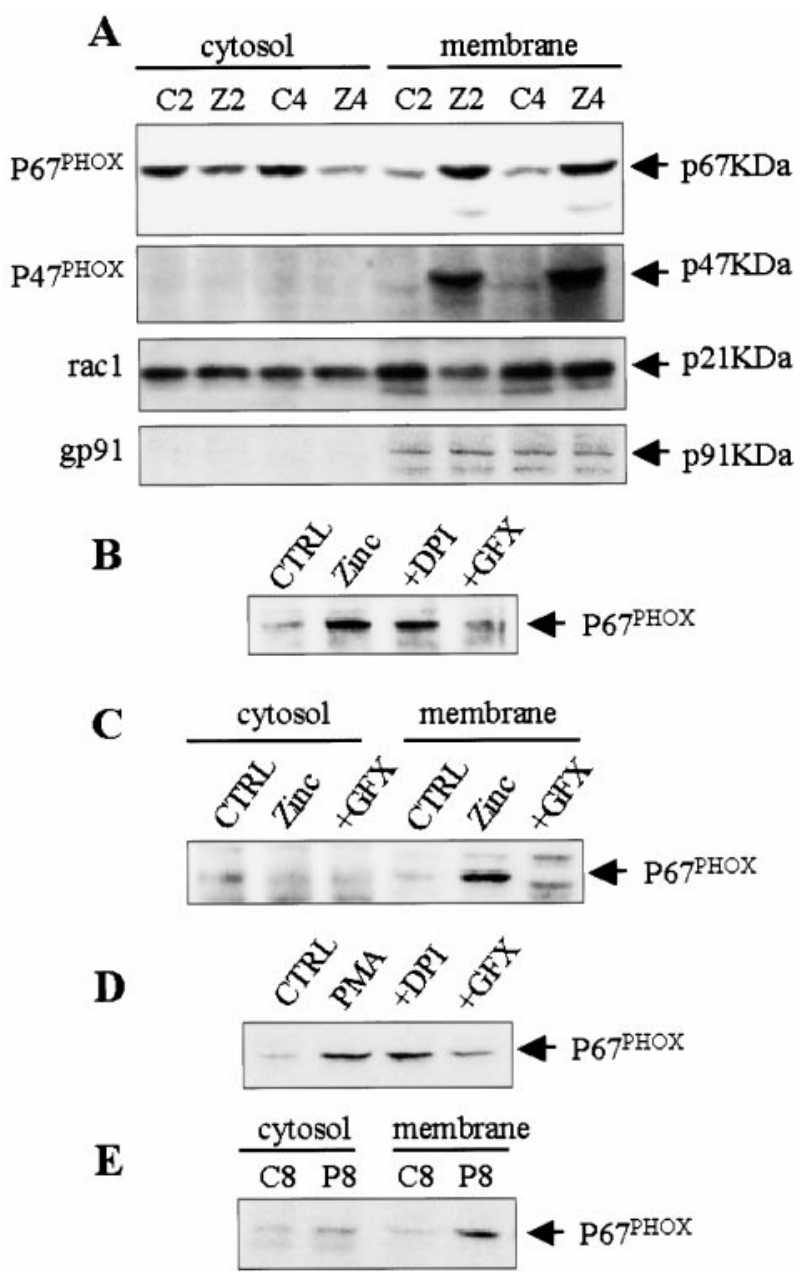

Figure 2. Mediation by PKC of translocation and induction of NADPH oxidase. $A$, Western blots for indicated subunits of NADPH oxidase in the cytosolic fraction and the membrane fraction of cortical cultures, 2 or 4 hr after sham wash or $15 \mathrm{~min}$ zinc exposure (representative of $5 \mathrm{ex}-$ periments). Whereas levels of rac1 in each fraction did not change much with zinc exposure, levels of p67 ${ }^{\text {PHOX }}$ appeared to be decreased in the cytosolic fraction and to be increased in the membrane fraction. Also, the membrane levels of $\mathrm{p} 47^{\mathrm{PHOX}}$ markedly increased upon zinc exposure. The level of the membrane-anchored subunit gp91 PHOX did not change much with zinc exposure. B, GF109203X (GFX) blocks NADPH oxidase induction by zinc. Western blots show levels of $\mathrm{p} 67^{\mathrm{PHOX}}$ in cortical cultures $4 \mathrm{hr}$ after sham wash $(C T R L)$ or $15 \mathrm{~min}$ exposure to 400 $\mu \mathrm{M}$ zinc without (Zinc) or with addition of DPI (100 nM) or GF109203X $(3 \mu \mathrm{M})$ during and continuously after the zinc exposure (representative of 4 experiments). GF109203X markedly attenuated the induction of p67 $7^{\text {PHOX }}$ by zinc, whereas DPI had no effect. $C$, GF109203X blocks NADPH oxidase activation by zinc. Western blots of the membrane fractions with anti-p67 $7^{\mathrm{PHOX}}$ antibody after $4 \mathrm{hr}$ exposure to $400 \mu \mathrm{M}$ zinc (representative of 5 experiments). Addition of $3 \mu \mathrm{M}$ GF109203X during and after the zinc exposure decreased membrane translocation of p67 ${ }^{\text {PHOX }}$ by zinc. $D$, PMA increases $067^{\text {PHOX }}$ levels. Western blots show levels of p67 $7^{\text {PHOX }}$ in sham-washed control or cultures after $8 \mathrm{hr}$ exposure to 200 nM PMA (representative of 4 experiments). The increase in p67 ${ }^{\text {PHOX }}$ levels by PMA was completely blocked by $3 \mu \mathrm{M}$ GF109203X, whereas $100 \mathrm{nM}$ DPI had little effect. $E$, PMA induces translocation of p67 $67^{\text {PHOX }}$. Membrane p67 $7^{\text {PHOX }}$ levels markedly increased with $8 \mathrm{hr}$ exposure to $200 \mathrm{nM}$ PMA (P8) (representative of 4 experiments) compared with cultures $8 \mathrm{hr}$ after sham wash $(C 8)$.

Measurement of ROS generation. Two independent methods were used to measure ROS production in cortical cultures. 2,7-Dichlorodihydrofluorescein diacetate (DCF) fluorescence was used to visualize intracellular superoxides (Greenlund et al., 1995). $\mathrm{H}_{2} \mathrm{O}_{2}$ accumulation in media was measured using the Amplex Red (10-acetyl-3,7-dihydroxyphenoxazine) Hydrogen Peroxide Assay kit (Molecular Probes, Eugene, OR).
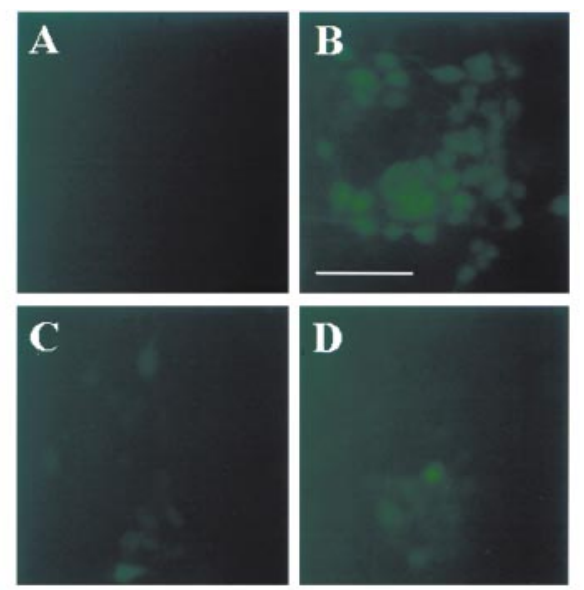

\section{E}

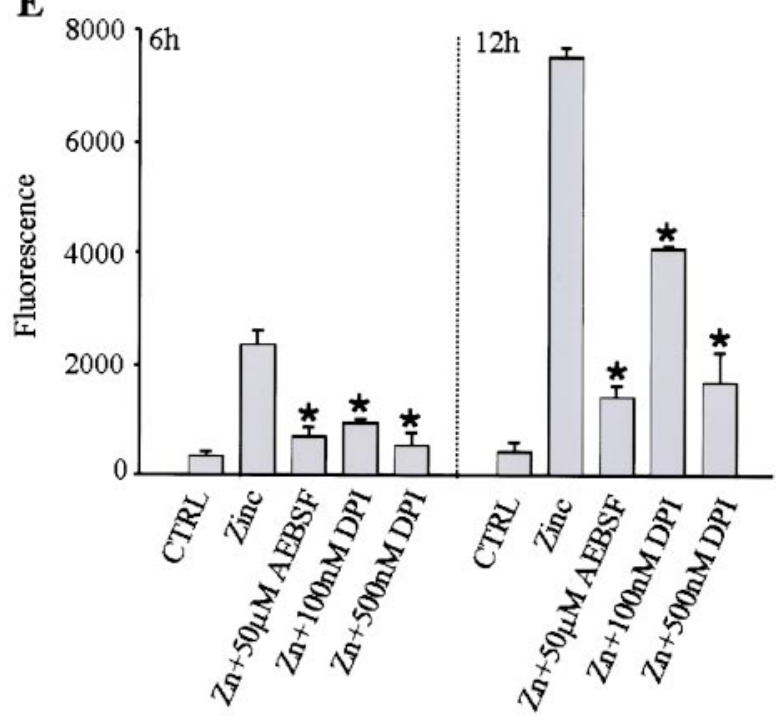

Figure 3. NADPH oxidase inhibitors attenuate ROS increases by zinc. $A-D$, DCF fluorescence in cortical cultures $3 \mathrm{hr}$ after sham wash $(A)$ or 15 min exposure to $400 \mu \mathrm{M}$ zinc without $(B)$ or with $(C)$ addition of $50 \mu \mathrm{M}$ AEBSF or $500 \mathrm{~nm}$ DPI $(D)$ during and after the zinc exposure. $E$, Levels of $\mathrm{H}_{2} \mathrm{O}_{2}$ in the bathing media of cortical cultures (mean + SEM; $n=3$ cultures) as estimated by the Amplex Red fluorimetry (arbitrary units of fluorescence). Media samples were obtained from sham-washed control cultures $(C T R L)$ or from cultures $6 \mathrm{hr}$ (left bars) or $12 \mathrm{hr}$ (right bars) after $15 \mathrm{~min}$ exposure to $400 \mu \mathrm{M}$ zinc without (Zinc) or with addition of AEBSF $(50 \mu \mathrm{M})$ or DPI $(100$ or $500 \mathrm{nM})$. Asterisks denote difference from Zinc $(p<0.05$; two-tailed $t$ test with Bonferroni correction for 3 comparisons). Scale bar, $100 \mu \mathrm{m}$.

\section{RESULTS}

\section{Expression and induction of NADPH oxidase in cultured cortical cells}

Subunits of NADPH oxidase were examined at the mRNA level by reverse transcription (RT)-PCR analysis. Whereas cortical cultures expressed low levels of mRNA for NADPH oxidase subunits (Fig. 1A), 15 min exposure to $400 \mu \mathrm{M}$ zinc substantially increased the mRNA levels for $\mathrm{p} 47^{\text {PHOX }}$, p67 $7^{\text {PHOX }}$, and gp91 ${ }^{\text {PHOX }}$ in cortical cultures with little change in that for p40 ${ }^{\text {PHOX }}$ (Fig. 1A). Western blot assay confirmed that zinc exposure increased the expression of both $\mathrm{p} 47^{\mathrm{PHOX}}$ and $\mathrm{p} 67^{\mathrm{PHOX}}$, beginning $\sim 30 \mathrm{~min}$ to $2 \mathrm{hr}$ after the exposure (Fig. 1B). Because mixed cortical cultures contain both neurons and astrocytes, we examined in which cell type NADPH oxidase expression increases in response to zinc exposure. Immunocytochemical stain- 


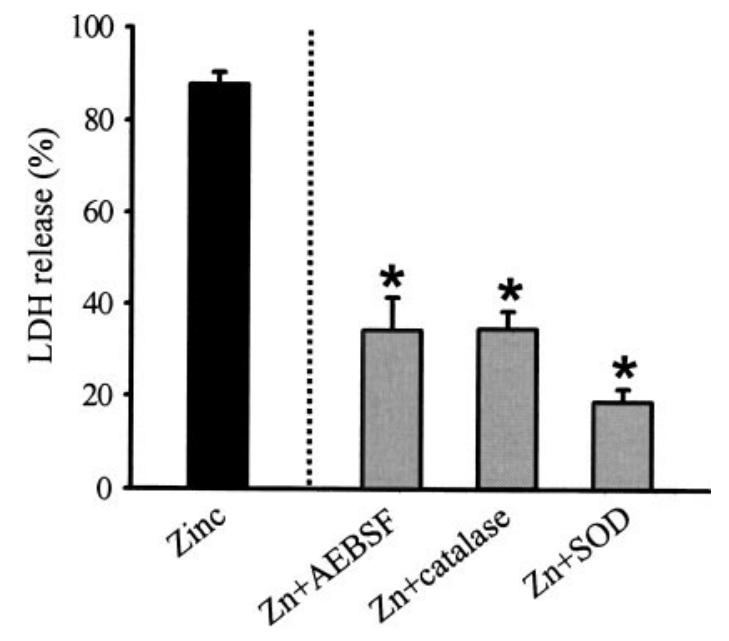

Figure 4. An NADPH oxidase inhibitor as well as $\mathrm{Cu} / \mathrm{Zn}-\mathrm{SOD}$ and catalase attenuate zinc neurotoxicity. $\mathrm{LDH}$ release in cortical cultures (mean $\pm \mathrm{SEM} ; n=3$ ) $24 \mathrm{hr}$ after 15 min exposure to $400 \mu \mathrm{M}$ zinc without (Zinc) or with addition of $50 \mu \mathrm{M}$ AEBSF, catalase $(300 \mathrm{mU} / \mathrm{ml})$, or $\mathrm{Cu} / \mathrm{Zn}$-SOD $(50 \mathrm{U} / \mathrm{ml})$. Asterisks denote difference from Zinc $(p<0.05$; two-tailed $t$ test with Bonferroni correction for 3 comparisons).

ing of neuron-rich cultures with neuron-specific anti-MAP2 antibody revealed that $>90 \%$ of cells were indeed neurons $(92.8 \pm$ $1.2 \%$ of Hoechst $33342^{+}$cells; $n=4$ ) (Fig. $1 C, E$ ). In control cultures, immunoreactivity to $67^{\text {PHOX }}$ was present at low level (Fig. 1D). However, $4 \mathrm{hr}$ after 15 min zinc exposure, immunoreactivity to p67 ${ }^{\text {PHOX }}$ markedly increased in the majority of $\mathrm{MAP}^{+}{ }^{+}$neurons (Fig. $1 F$ ). On the other hand, in astrocyte-rich cultures, almost all cells exhibited immunoreactivity to antiGFAP antibody $\left(97.8 \pm 1.4 \%\right.$ of Hoechst $33342^{+}$cells; $\left.n=4\right)$ (Fig. 1G,I). Again, baseline expression of p67 ${ }^{\text {PHOX }}$ appeared quite low in astrocytes (Fig. $1 H$ ). However, exposure to zinc substantially increased p67 ${ }^{\text {PHOX }}$ levels in most astrocytes (Fig. $1 J)$. Hence, zinc exposure increased $\mathrm{p} 67^{\text {PHOX }}$ expression in both neurons and astrocytes.

\section{PKC mediates induction and membrane translocation of NADPH oxidase}

Next, we examined whether zinc exposure leads to the functional activation of NADPH oxidase. The necessary step for NADPH oxidase activation is the translocation of cytosolic subunits, including $\mathrm{p} 47^{\text {PHOX }}$ and p $67^{\text {PHOX }}$, to the membrane (Leusen et al., 1996; Han et al., 1998; Johnson et al., 1998). Consistent with the membrane translocation, at 2 and $4 \mathrm{hr}$ after $15 \mathrm{~min}$ zinc exposure, levels of $\mathrm{p} 47^{\text {PHOX }}$ and $\mathrm{p} 67^{\text {PHOX }}$ in the membrane fraction markedly increased (Fig. $2 A$ ). Because p $47^{\text {PHOX }}$ was barely detectable in the cytosol in both control and zinc-exposed cultures (Fig. 2A), markedly increased $\mathrm{p} 47^{\mathrm{PHOX}}$ in membrane of zinc-exposed cells may signify the fairly rapid recruitment of newly synthesized protein to the membrane. It is well known in phagocytic cells that levels and activity of NADPH oxidase are regulated by protein kinase C (Heinecke et al., 1990; Benna et al., 1997; Reeves et al., 1999). Because zinc activates PKC (Murakami et al., 1987; Csermely et al., 1988; Noh et al., 1999), we examined whether the effect of zinc on NADPH oxidase induction and activation is mediated by PKC. Indeed, a selective PKC inhibitor, GF109203X, completely blocked the induction of $\mathrm{p} 67^{\mathrm{PHOX}}$ by zinc, whereas a selective inhibitor of NADPH oxidase, diphenyleneiodonium (DPI), did not (Fig. 2B). Furthermore, the membrane translocation of p67 ${ }^{\text {PHOX }}$ by zinc was also inhibited by GF109203X (Fig.
$2 C$ ). Conversely, addition of a PKC activator, PMA, alone was sufficient for the induction and translocation of p67 PHOX $_{\text {in cor- }}$ tical cultures (Fig. 2D,E). Induction of NADPH oxidase by PMA was blocked by GF109203X but not by DPI (Fig. 2D).

\section{NADPH oxidase contributes to ROS generation induced by zinc}

We have shown previously that zinc exposure leads to the activation of $\mathrm{PKC}$, which leads to increases in superoxide generation in cultured cortical neurons (Noh et al., 1999). Having demonstrated that PKC mediates the induction and activation of NADPH oxidase by zinc, we examined whether NADPH oxidase really contributes to superoxide generation after zinc exposure. Exposure of mixed cortical cultures to zinc markedly increased cellular DCF fluorescence (Fig. $3 B$ ), an indicator for superoxide, compared with controls (Fig. $3 A$ ). NADPH oxidase inhibitors 4-(2-aminoethyl)-benzenesulfonyl fluoride (AEBSF) and DPI (Diatchuk et al., 1997; Li and Trush, 1998) markedly reduced the DCF fluorescence (Fig. 3C,D). This finding was confirmed independently by the Amplex Red fluorimetry assay; gradual $\mathrm{H}_{2} \mathrm{O}_{2}$ accumulation in the media was observed at 6 and $12 \mathrm{hr}$ after zinc exposure. NADPH oxidase inhibitors AEBSF and DPI markedly reduced $\mathrm{H}_{2} \mathrm{O}_{2}$ buildup in the media of zinc-exposed cultures at both times (Fig. 3E).

\section{NADPH oxidase contributes to oxidative neuronal death induced by zinc}

The next question was whether ROS generated by NADPH oxidase contributes to zinc-induced neuronal death. Neurons exhibited marked cell body swelling several hours after $15 \mathrm{~min}$ zinc exposure, followed by release of $\mathrm{LDH}$ into the bathing medium (Fig. 4), as described previously (Kim et al., 1999). Addition of AEBSF significantly reduced neuronal death and resultant LDH release induced by the zinc exposure (Fig. 4); we could not use another NADPH oxidase inhibitor, DPI, because even at low concentrations (100 nM), it had significant cytotoxicity (data not shown). Addition of $\mathrm{Cu} / \mathrm{Zn}$-superoxide dismutase (SOD), which lowers superoxide levels, also attenuated zincmediated neuronal death (Fig. 4).

\section{DISCUSSION}

Although oxidative stress has been proposed as one of the major mechanisms of ischemic brain injury (Uyama et al., 1992; Chan et al., 1998), detailed information about the cascades connecting ischemic events to increased ROS generation is currently lacking. Combined with the evidence that zinc overload is a key mechanism of ischemic neuronal death (Choi and Koh, 1998), the present study suggests that the induction and activation of NADPH oxidase in intrinsic brain cells by zinc overload may play such a role in ischemic brain injury.

Although the presence of NADPH oxidase in sympathetic ganglion neurons has raised the possibility that other neurons may also express NADPH oxidase (Tammariello et al., 2000), functional NADPH oxidase expression in central neurons and astrocytes has not been directly documented. The present study has demonstrated that NADPH oxidase subunits, albeit at low levels, are indeed expressed in central neurons and astrocytes. More interestingly, certain subunits of NADPH oxidase were rapidly induced after injurious zinc exposure. Furthermore, in addition to being induced, NADPH oxidase appears to be functionally activated after zinc exposure.

The next question was which event links between zinc overload and NADPH oxidase induction-activation. Whereas several 
known activators of NADPH oxidase are known, we focused on PKC in this study, because zinc has been shown to directly activate PKC (Noh et al., 1999). Supporting this possibility, addition of a selective PKC inhibitor blocked the induction and membrane translocation of p67 ${ }^{\text {PHOX }}$ after zinc exposure. Conversely, addition of a PKC activator (PMA) was sufficient to induce and activate NADPH oxidase. Together, these results suggest a critical role for PKC in both induction and activation of NADPH oxidase by zinc.

In the present study done in cortical cultures, the contribution by NADPH oxidase to zinc-induced oxidative injury was directly supported by the findings that inhibitors of NADPH oxidase attenuate both superoxide generation and neuronal death after zinc exposure. This again supports the idea that oxidative stress is a significant mechanism of zinc overload neurotoxicity. Consistently, $\mathrm{Cu} / \mathrm{Zn}-\mathrm{SOD}$ and catalase, enzymes reducing free radical levels, significantly attenuated zinc-induced neuronal death. Together, the present results suggest that, in brain injury conditions in which zinc neurotoxicity contributes, PKC activation and the resultant NADPH oxidase induction-activation may play a significant role in causing oxidative neuronal injury. Consistent with this idea, the PKC inhibitor staurosporine and antioxidative measures have been shown to attenuate the death of CA1 neurons after transient ischemia (Hara et al., 1990; Tagami et al., 1999; Sheng et al., 2000) in which zinc neurotoxicity may play a significant role (Koh et al., 1996).

The present study, for the first time, has demonstrated the expression and activation of NAPDH oxidase in cultured cortical neurons and astrocytes are under control of PKC, which is activated by zinc influx. Additional studies seem warranted to elucidate the potential role for NADPH oxidase in oxidative injury in vivo as well, because oxidative stress is likely a common mechanism for cellular damage in various neurological diseases.

\section{REFERENCES}

Babior BM (1999) NADPH oxidase: an update. Blood 93:1464-1476.

Benna JE, Dang PM, Gaudry M, Fay M, Morel F, Hakim J, GougerotPocidalo MA (1997) Phosphorylation of the respiratory burst oxidase subunit p67(phox) during human neutrophil activation. Regulation by protein kinase $\mathrm{C}$-dependent and independent pathways. J Biol Chem 272:17204-17208.

Chan PH, Kawase M, Murakami K, Chen SF, Li Y, Calagui B, Reola L, Carlson E, Epstein CJ (1998) Overexpression of SOD1 in transgenic rats protects vulnerable neurons against ischemic damage after global cerebral ischemia and reperfusion. J Neurosci 18:8292-8299.

Choi DW, Koh JY (1998) Zinc and brain injury. Annu Rev Neurosci 21:347-375.

Csermely P, Szamel M, Resch K, Somogyi J (1988) Zinc can increase the activity of protein kinase $\mathrm{C}$ and contributes to its binding to plasma membranes in T lymphocytes. J Biol Chem 263:6487-6490.

DeLeo FR, Quinn MT (1996) Assembly of the phagocyte NADPH oxidase: molecular interaction of oxidase proteins. J Leukoc Biol 60:677-691.

Diatchuk V, Lotan O, Koshkin V, Wikstroem P, Pick E (1997) Inhibition of NADPH oxidase activation by 4-(2-aminoethyl)-benzenesulfonyl fluoride and related compounds. J Biol Chem 272:13292-13301.

Frederickson CJ, Hernandez MD, McGinty JF (1989) Translocation of zinc may contribute to seizure-induced death of neurons. Brain Res 480:317-321.

Fukui T, Lassegue B, Kai H, Alexander RW, Griendling KK (1995) Cytochrome b-558 alpha-subunit cloning and expression in rat aortic smooth muscle cells. Biochim Biophys Acta 1231:215-219.

Greenlund LJ, Deckwerth TL, Johnson Jr EM (1995) Superoxide dismutase delays neuronal apoptosis: a role for reactive oxygen species in programmed neuronal death. Neuron 14:303-315.

Han CH, Freeman JL, Lee T, Motalebi SA, Lambeth JD (1998) Regulation of the neutrophil respiratory burst oxidase. Identification of an activation domain in p67(phox). J Biol Chem 273:16663-16668.

Hara H, Onodera H, Yoshidomi M, Matsuda Y, Kogure K (1990) Staurosporine, a novel protein kinase $\mathrm{C}$ inhibitor, prevents postischemic neuronal damage in the gerbil and rat. J Cereb Blood Flow Metab 10:646-653.
Heinecke JW, Meier KE, Lorenzen JA, Shapiro BM (1990) A specific requirement for protein kinase $\mathrm{C}$ in activation of the respiratory burst oxidase of fertilization. J Biol Chem 265:7717-7720.

Johnson JL, Park JW, Benna JE, Faust LP, Inanami O, Babior BM (1998) Activation of p47(PHOX), a cytosolic subunit of the leukocyte NADPH oxidase. Phosphorylation of ser-359 or ser-370 precedes phosphorylation at other sites and is required for activity. $\mathrm{J}$ Biol Chem 273:35147-35152.

Jones SA, Hancock JT, Jones OT, Neubauer A, Topley N (1995) The expression of NADPH oxidase components in human glomerular mesangial cells: detection of protein and mRNA for p47phox, p67phox, and p22phox. J Am Soc Nephrol 5:1483-1491.

Jones SA, O’Donnell VB, Wood JD, Broughton JP, Hughes EJ, Jones OT (1996) Expression of phagocyte NADPH oxidase components in human endothelial cells. Am J Physiol 271:H1626-H1634.

Kim YH, Kim EY, Gwag BJ, Sohn S, Koh JY (1999) Zinc-induced cortical neuronal death with features of apoptosis and necrosis: mediation by free radicals. Neuroscience 89:175-182.

Koh JY, Choi DW (1987) Quantitative determination of glutamate mediated cortical neuronal injury in cell culture by lactate dehydrogenase efflux assay. J Neurosci Methods 20:83-90.

Koh JY, Suh SW, Gwag BJ, He YY, Hsu CY, Choi DW (1996) The role of zinc in selective neuronal death after transient global cerebral ischemia. Science 272:1013-1016.

Leusen JH, Verhoeven AJ, Roos D (1996) Interactions between the components of the human NADPH oxidase: intrigues in the phox family. J Lab Clin Med 128:461-476.

Li Y, Trush MA (1998) Diphenyleneiodonium, an NAD(P)H oxidase inhibitor, also potently inhibits mitochondrial reactive oxygen species production. Biochem Biophys Res Commun 253:295-299.

Lobner D, Gottron F, Ying H, Tian M, Dugan LL (1997) Zinc-induced neuronal apoptosis or necrosis in cortical cell culture. Soc Neurosci Abstr 23:2255.

Manev H, Kharlamov E, Uz T, Mason RP, Cagnoli CM (1997) Characterization of zinc-induced neuronal death in primary cultures of rat cerebellar granule cells. Exp Neurol 146:171-178.

Murakami K, Whiteley MK, Routtenberg A (1987) Regulation of protein kinase $\mathrm{C}$ activity by cooperative interaction of $\mathrm{Zn}^{2+}$ and $\mathrm{Ca}^{2+}$. J Biol Chem 262:13902-13906.

Noh KM, Kim YH, Koh JY (1999) Mediation by membrane protein kinase $\mathrm{C}$ of zinc-induced oxidative neuronal injury in mouse cortical cultures. J Neurochem 72:1609-1616.

Ozaki M, Deshpande SS, Angkeow P, Bellan J, Lowenstein CJ, Dinauer MC, Goldschmidt-Clermont PJ, Irani K (2000) Inhibition of the Rac1 GTPase protects against nonlethal ischemia/reperfusion-induced necrosis and apoptosis in vivo. FASEB J 14:418-429.

Reeves EP, Dekker LV, Forbes LV, Wientjes FB, Grogan A, Pappin DJ, Segal AW (1999) Direct interaction between $\mathrm{p} 47$ phox and protein kinase $\mathrm{C}$ : evidence for targeting of protein kinase $\mathrm{C}$ by $\mathrm{p} 47 \mathrm{phox}$ in neutrophils. Biochem J 344:859-866.

Sensi SL, Yin HZ, Carriedo SG, Rao SS, Weiss JH (1999) Preferential $\mathrm{Zn}^{2+}$ influx through $\mathrm{Ca}^{2+}$-permeable AMPA/kainate channels triggers prolonged mitochondrial superoxide production. Proc Natl Acad Sci USA 96:2414-2419.

Sheline CT, Choi DW (1998) Neuronal death in cultured murine cortical cells is induced by inhibition of GAPDH and triosephosphate isomerase. Neurobiol Dis 5:47-54.

Sheline CT, Behrens MM, Choi DW (2000) Zinc-induced cortical neuronal death: contribution of energy failure attributable to loss of NAD ${ }^{+}$ and inhibition of glycolysis. J Neurosci 20:3139-3146.

Sheng H, Kudo M, Mackensen GB, Pearlstein RD, Krapo JD, Warner DS (2000) Mice overexpressing extracellular superoxide dismutase have increased resistance to global cerebral ischemia. Exp Neurol 163:392-398.

Suh SW, Chen JW, Motamedi M, Bell B, Listiak K, Pons NF, Danscher G, Frederickson CJ (2000) Evidence that synaptically-released zinc contributes to neuronal injury after traumatic brain injury. Brain Res 852:268-273.

Tagami M, Ikeda K, Yamagata K, Nara Y, Fujino H, Kubota A, Numano F, Yamori Y (1999) Vitamin E prevents apoptosis in hippocampal neurons caused by cerebral ischemia and reperfusion in stroke-prone spontaneously hypertensive rats. Lab Invest 79:609-615.

Tammariello SP, Quinn MT, Estus S (2000) NADPH oxidase contributes directly to oxidative stress apoptosis in nerve growth factordeprived sympathetic neurons J Neurosci 20:RC53.

Thannickal VJ, Fanburg BL (1995) Activation of an $\mathrm{H}_{2} \mathrm{O}_{2}$-generating NADH oxidase in human lung fibroblasts by transforming growth factor beta 1. J Biol Chem 270:30334-30338.

Tonder N, Johansen FF, Frederickson CJ, Zimmer J, Diemer NH (1990) Possible role of zinc in the selective degeneration of dentate hilar neurons after cerebral ischemia in the adult rat. Neurosci Lett 109:247-252.

Uyama O, Matsuyama T, Michishita H, Nakamura H, Sugita M (1992) Protective effects of human recombinant superoxide dismutase on transient ischemic injury of CA1 neurons in gerbils. Stroke 23:75-81. 